\title{
Intelligent robotics - misconceptions, current trends, and opportunities
}

\author{
Clarence W. de Silva \\ Department of Mechanical Engineering, University of British Columbia, Vancouver BC V6T 1Z4, Canada.
}

Correspondence to: Prof. Clarence W. de Silva, Department of Mechanical Engineering, University of British Columbia, 6250 Applied Science Lane, Vancouver BC V6T 1Z4, Canada. E-mail: desilva@mech.ubc.ca

How to cite this article: de Silva CW. Intelligent robotics - misconceptions, current trends, and opportunities. Intell Robot 2021;1(1):3-17. https://dx.doi.org/10.20517/ir.2021.01

Received: 25 Jul 2021 First Decision: 21 Jul 2021 Revised: 24 Jul 2021 Accepted: 28 Jul 2021 Published: 11 Oct 2021

Academic Editor: Simon X. Yang Copy Editor: Xi-Jun Chen Production Editor: Xi-Jun Chen

\begin{abstract}
The concepts of "Robots" have been of interest to humans from historical times, initially with the desire to create "artificial slaves". Since the technology was not developing to keep up with the "dreams", initially, Robotics was primarily of entertainment value, relegated to plays, movies, stories, etc. The practical applications started in the late 1950s and the 1960s with the development of programmable devices for factories and assembly lines as flexible automation. However, since the expectations were not adequately realized, the general enthusiasm and funding for Robotics subsided to some extent. With subsequent research, developments, and curricular enhancement in Engineering and Computer Science and the resurgence of Artificial Intelligence, particularly machine learning, Robotics has found numerous practical applications today, in industry, medicine, household, the service sector, and the general society. Important developments and practical strides are being made, particularly in Soft Robotics, Mobile Robotics (Aerial - drones, Underwater, Ground-based - autonomous vehicles in particular), Swarm Robotics, Homecare, Surgery, Assistive Devices, and Active Prosthesis. This perspective paper starts with a brief history of Robotics while indicating some associated myths and unfair expectations. Next, it will outline key developments in the area. In particular, some important practical applications of Intelligent Robotics, as developed by groups worldwide, including the Industrial Automation Laboratory at the University of British Columbia, headed by the author, are indicated. Finally, some misconceptions and shortcomings concerning Intelligent Robotics are pointed out. The main shortcomings concern the mechanical capabilities and the nature of intelligence. The paper concludes by mentioning future trends and key opportunities available in Intelligent Robotics for both developed and developing counties.
\end{abstract}


Keywords: Robotics, characteristics of intelligence, machine learning, shortcomings of intelligent robotics, technical needs, opportunities in intelligent robotics

\section{INTRODUCTION}

Commonly, a robot is considered a machine that can perform work or actions normally performed by humans, automatically or by remote control - Teleoperation. The key features of this definition are the presence of: (1) Mechanical Structure (Machine); (2) Sensors; (3) Actuators (or Effectors); and (4) Controller (or Computer), which is the brain or the decision-maker of the robot. Essentially, a digital computer serves as this "brain" of the robot, and it has to be programmed to carry out its actions. Therefore, the "intelligence" of a robot needs to be incorporated there. As well, there have to be sensors to monitor the operation. The sensory data are processed by the computer to determine the underlying situations and the suitable robotic actions.

The concepts of "Robots" have been of interest to humans from historical times, initially with the desire to create "artificial slaves". The term "Robot" was introduced in the popular media, well before a physical robot became a reality. In 1920, Czech writer Karel Capek first introduced the term in his play "RUR" or "Rossum's Universal Robots". There, it was just a figment of his imagination. Again, in 1942, the Russianborn American science-fiction writer and Boston University Professor Isaac Asimov introduced the term in his fiction. Notably, Asimov was one of the "Big Three" in science fiction. The other two were the Sri Lankabased late Sir Arthur C. Clarke and Robert A. Heinlein. We know that many predictions of Clarke and Asimov have come true today. A device resembling a humanoid robot was designed and built by the ingenious Leonardo Da Vinci in 1945. It could mechanically move arms, head, and jaw but was not a true robot in today's definition. The first true robot arm, the Unimate, was designed by the American inventor George Devol in collaboration with Joseph Engelberger, who is often called the "Father of Robotics". This robot was used in a General Motors (automotive) plant for its manufacturing operations in 1960. It had a primitive digital computer as its brain and used motion sensors and also dc motors as the actuators ${ }^{[1]}$.

Many different types of robots have been developed and put into operation since. Some that we see in the popular media are, however, computer animations rather than "intelligent" robots. Since the technology was not developing to keep up with the "dreams", initially, Robotics was primarily of entertainment value, relegated to plays, movies, stories, etc. The practical applications started in the late 1950s and the 1960s with the development of programmable devices for factories and assembly lines as flexible automation. As an example of the application of Robotics in flexible automation, consider the welding robots in an automotive plant ${ }^{[2]}$. Here, the vehicle model that is being manufactured can be changed very easily and quickly, simply by changing the program. However, the operation itself is not fast, albeit quite complex. However, since the expectations were not adequately realized, the general enthusiasm and funding for Robotics subsided to some extent. With subsequent research, development, and curricular enhancement in Engineering and Computer Science and the resurgence of Artificial Intelligence (AI), particularly machine learning, Intelligent Robotics has found numerous practical applications today in industry, medicine, household, and the general society. Important developments and practical strides are being made, particularly in Soft Robotics, Mobile Robotics (Aerial - drones, Underwater, and Ground-based - autonomous vehicles in particular), Swarm Robotics, Homecare, Surgery, Assistive Devices, and Active Prosthesis.

\section{THE STATE-OF-THE-ART}

Many different types of robots are available today. A robot that has a human-like body structure is called a "humanoid". An example is the Honda Asimo ${ }^{[3]}$. However, a robot need not look like a human. An 
Unmanned Naval Vehicle that we developed in Figure 1 in our laboratory (Industrial Automation Laboratory or IAL at the University of British Columbia or UBC) is indeed a robot. It has a programmable propelling platform with multiple sensors (to measure the $\mathrm{pH}$ value, dissolved oxygen, electrical conductivity, temperature, and the oxidation-reduction potential of water). It is able to autonomously navigate in a water body and map out the quality of the water in a particular region, which can be used to provide warnings to the users, determine the source of pollution or contamination, and also suggest to the relevant authorities suitable corrective actions $s^{[4]}$.

Unmanned aerial vehicles (UAVs) or drones are other types of mobile robots. A UAV that is used in our laboratory is shown in Figure 2. A similar UAV is used in our collaborative project in environmental monitoring and field mapping ${ }^{[5]}$. Due to their capabilities of autonomous navigation in extensive and traditionally unreachable regions and advanced sensing (such as optical and thermal imaging and laser scanning), UAVs are replacing costly, hazardous, and manual ways of inspection and surveillance in various applications. For example, when compared with the manual and ground-based approaches, UAVs are able to capture more useful and detailed information of petrochemical operations, including wellheads, pipelines, boiler systems, tanks, refineries, and furnaces, in a consistent, continuous, accurate, fast, and repeated manner, even in environments where human operators find it time-consuming, difficult and hazardous to function. The data/information collected in this manner can be further processed in a groundbased control platform and used to localize faults and malfunctions in the facility. Based on that information, a UAV is able to deploy the needed equipment, including robotic devices, to that location for detailed diagnosis and possible correction of the problem.

As another example, consider the smart prosthetic $\operatorname{limb}^{[6]}$ shown in Figure 3. It is able to adapt to different users and conditions of the terrain intelligently. Also, it uses expectational knowledge during walking, for example, to decide whether to step over an obstacle or to avoid it.

\subsection{Common myths of robotics}

There have been many myths, misnomers, and misunderstandings surrounding robots. Some are:

1. Robots have capabilities that equate to or exceed those of humans. This is not true. The intelligence of today's robots does not exceed even that of a dog! It is inconceivable that robot intelligence can reach that of human intelligence.

2. Robots will steal our spouses, and fight wars, and defeat us. This is very unrealistic and will never happen! 3. Robots will create mass unemployment: people said this concerning the first Industrial Revolution as well. However, in the end, the industrial revolution (and industrial automation) gave the workers more free time (including the five-day workweek), moved them away from hazardous and difficult work environments, improved the general quality of life, and created higher-paying and more challenging employment (for our friends and relative, if not for us).

While many positive things can be said about robotization, the reasons for the underlying misunderstandings are several. They include fantasy, movies, and popular/social media, unrealistic expectations by the robot enthusiasts, slow developments in the field, inadequate expertise and capabilities for the necessary developments, the lack of necessary technologies (mechanical, electronic, and computer science, etc.) to achieve the goals, weak collaboration in the beginning (mainly among the electrical and mechanical engineers, and computer scientists, but this culture has been changed with the establishment of leading robotics institutes); and the lack of adequate "robotic intelligence" for various autonomous operations. See the related video interview of the author ${ }^{[7]}$. 

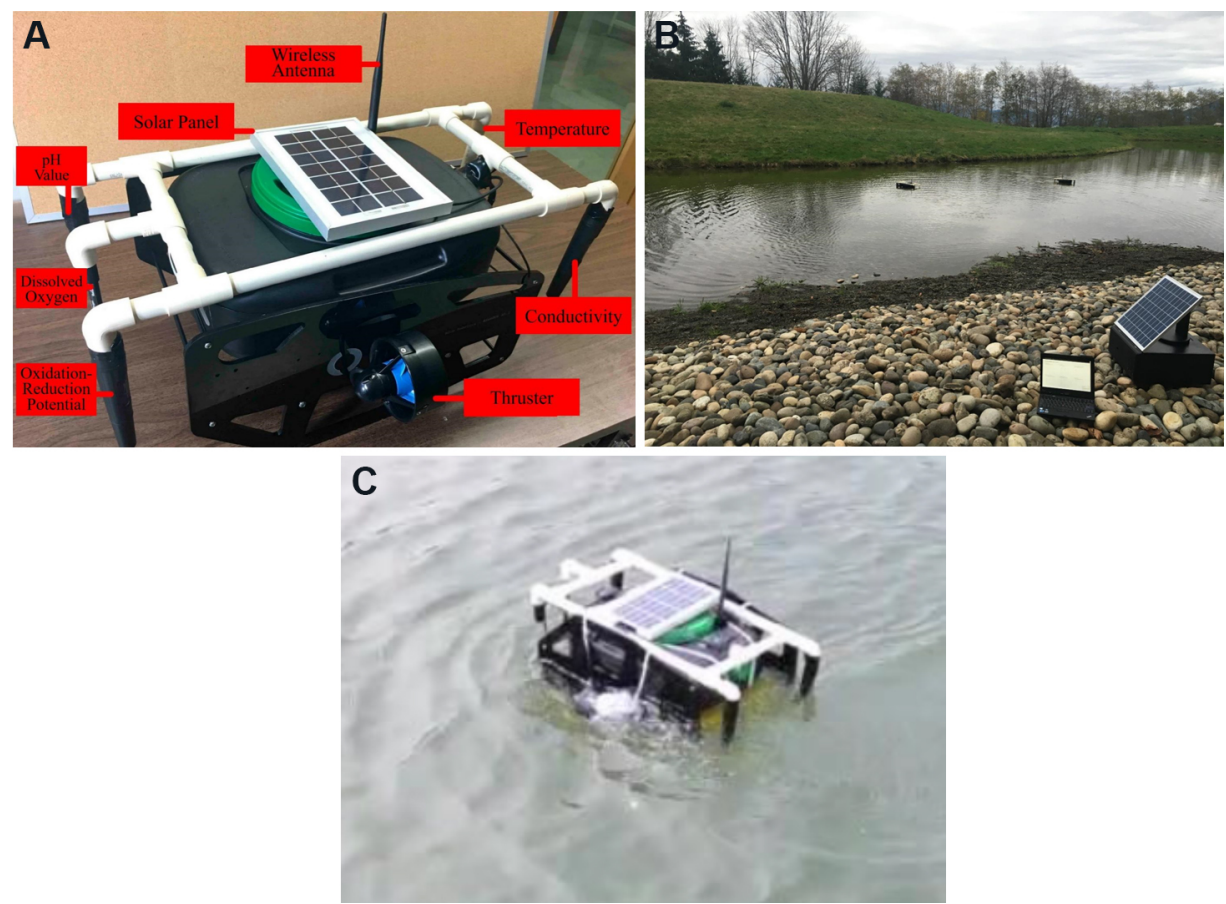

Figure 1. An autonomous naval multi-sensor module that monitors and maps the quality of water. (A) Components of the Unmanned Naval Vehicle; (B) Multi-module deployment in Vancouver; (C) Deployment in a river in India.

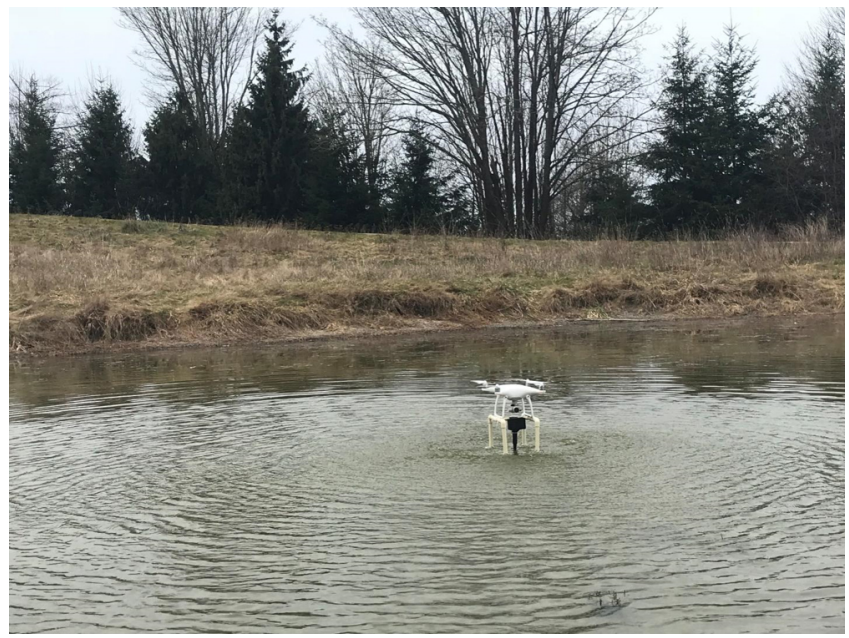

Figure 2. An unmanned aerial vehicle that is used in our laboratory.

\section{SHORTCOMINGS AND NEEDS}

What the engineers and technologists reasonably expect in Robotics has not been realized yet. The common capabilities of the existing robots include navigation with obstacle avoidance (SLAM - Simultaneous Localization and Mapping), visual and verbal communication with humans, operation of some appliances, grasping and carrying of objects (including conformable grasping and tactile sensing), multi-robot cooperation, and haptic teleoperation (with force feedback). However, some obvious shortcomings of today's robots include poor human-like interaction (and poor interaction with humans), slow speed, poor dexterity, and the sequential nature in grasping and handling of objects (e.g., the robotic hand slowly moves 


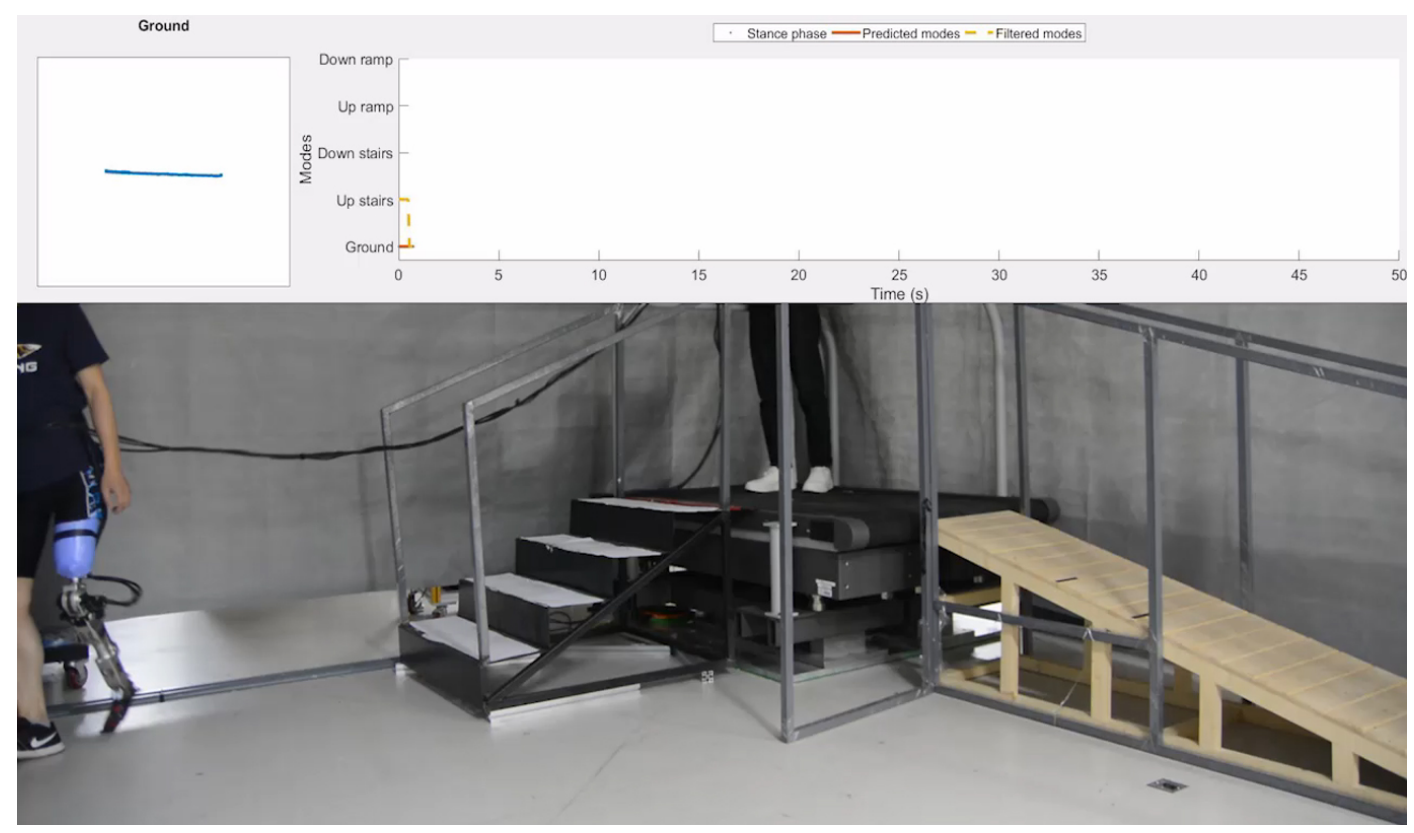

Figure 3. A smart prosthetic limb.

to the object and subsequently the fingers are closed around the object. Instead, in the case of a human, they observe the object to be grasped and simultaneously move the hand and the body in coordination until the object is reached and the grasping is completed. Also, a human can easily sense possible slipping of the object before it occurs), possible safety problems for humans (due to the shortcomings of the current robotic mechanical components and control), sensory limitations (particularly related to smell and taste, or chemical and biological sensing; transparency in teleoperation), limited mechanical capability (in dexterity, flexibility, adaptability, etc., unlike humans), and limited robotic intelligence. Concerning the safety in human-robot interaction, robot-inflicted injuries include accidents involving: sharp objects and tools, large forces, fast motions and quick changes of magnitude and direction, and malfunctions in the robotic equipment.

The autonomous operation (i.e., operating on its own, without outside help) of robots is essential in Intelligent Robotics. Some needs for this autonomy are greater robotic intelligence (better learning; operating in dynamic, partially structured, and partially known environments; the capability of enhanced characteristics of intelligence); greater accuracy, speed, dexterity, etc.; increased safety (better object handling, accident/obstacle avoidance, etc.); more human-friendly and human-like communication and operation; and the redesigning of household appliances for easy operation by robots (and humans).

Consider the required basic caregiver tasks (of humans), for example, verbal and visual communication; assistance for movement/mobility; identifying, grasping, and handling of needed objects properly and safely; safe and quick assistance in the mobility of the care-receiver, in the presence of obstacles (both static and dynamic such as furniture, appliances, other humans and pets); monitoring of objects and the environment for carrying out the caregiver tasks (under normal and emergencies); operating household appliances; and the provision of assistive devices. Similar operational requirements from a robotic caregiver include faster yet safe operation; human-friendly and human-like interaction and communication; autonomous assistance for $24 \mathrm{~h}$, routine and basic care (mobility, bathing, dressing, toileting, meal preparation, providing medicine, etc.); effective monitoring and detection of emergencies; and adequate 
emergency assistance (possibly incorporating remote monitoring, teleoperation, etc.) until regular help arrives. In this context, some needs in haptic teleoperation (teleoperation with feedback from the slave robot to the human master) are improvements to autonomous robotics as in non-teleoperation situations; improved transparency (better/faster tactile/visual/auditory feedback to the remote human operator, for realistic creation of remote presence); stability under (and compensation for) time delays (which are common in teleoperation ${ }^{[8]}$; human-like manipulation; improved design and control (for accuracy, speed, robustness, reliability, and safety); and $3 \mathrm{D}$ virtual reality for the remote operator (for improved transparency).

\subsection{Possible directions of advancement}

The technology focus may be directed on several aspects to improve the state-of-the-art of Intelligent Robotics. They include autonomy, improved learning and intelligence (for autonomous operation); selfawareness for robots (i.e., knowing the own capabilities of the robot); improved dexterity of handling (e.g., compliant grasping, parallel not sequential, and incoordination; providing the adequate degrees of freedom for manipulation of the handled object); improved robot-human interaction (in particular, working "with" a human rather than working "for" a human); improved speed, stability, robustness, reliability, and safety; improved sensing (particularly, chemical and biological sensing; transparency of remote operations; dynamic sensor networks; intelligent sensor fusion); and significant improvement of the "mechanical" capabilities. Note particularly the capabilities that require "intelligence", software, mechanical capabilities, and instrumentation.

In this context, a question can be posed whether to direct much effort in developing universal robots that have unlimited capabilities and functions, which will, of course, be very costly and complex as well. In other words, should the focus be directed on the development of very complex and costly multi-purpose robots or use existing single-purpose robots cooperatively? In fact, it is not wise to put much effort into the development of complex and costly robots with numerous features and multi-function capabilities. As a scenario, consider the use of existing low-cost robots that have been developed for just one specific task ( e.g., security, human assistance, and guidance, street cleaning). If an emergency occurs (e.g., an explosion), they may be called upon to join (if available) in cooperation, for example, put together simple devices and assist in the situation (e.g., evacuation of the injured).

\section{ROBOTIC INTELLIGENCE}

The importance of intelligence in Robotics is quite clear. In particular, intelligence is essential for the autonomous operation of a robot. In fact, the realization of expectations (including some fantasies?) of intelligent robotics depends on improved robotic intelligence and similarly improved mechanical capability. Today's robots do not have even "primitive" human intelligence. Without significantly improved intelligence, robots cannot achieve human-like capabilities; for example, emotions, common sense, and inventiveness are rather farfetched! Improved intelligence renders the robots acquire some characteristics of human intelligence. A robot may be "trained" for a specific task (through methodologies of machine learning), but this is not the same as developing the robotic brain to reach the nature and capabilities of a human brain, at least at a very basic level. It is simply "fear-mongering" to say that the future robots will be a danger to humankind because of their high level of intelligence.

Primarily, robots improve their intelligence through learning, and the foundation of AI is indeed machine learning. Some claim that since a chess-playing computer has defeated a human champion, it is possible that intelligent robots will defeat humans in many human-centered activities. Here we have to realize that the capabilities of a robot depend on their mechanical capabilities and the control program (or brain), which is 
developed by humans. It is true that due to learning, the particular robot intelligence (the decision-making ability related to the learned knowledge) improves. Unlike humans, whose level of intelligence can depreciate for many reasons (physiology, lack of practice, new knowledge, new and complex environments, etc.), machine learning will always improve robotic intelligence. This means a chess-playing robot will continuously improve its skills through learning (practice) and can thereby defeat a chess champion. However, we have to realize that such intelligence is provided to robots by humans through control programs. That program will never be able to acquire all the characteristics of a human brain. For instance, we may question whether a chess-playing robot can also perform other tasks (e.g., caring for an elderly, carrying out medical surgery) unless specifically developed and trained for such activities and has the needed mechanical capabilities. Also, can a robot ever acquire such characteristics as common sense or emotions that are possessed by a human, in the same manner as in a human brain?

Humans develop robots, and we program their controllers (brains). We can set limits, checks and balances, regulations, and guidelines as we wish. We should collaborate with social scientists and develop proper guidelines and regulations for the development and the safety and ethical use of robots. Since a proper ethical evaluation and certification are essential for any technology that is used by humans, this should properly adhere for robots as well. In medical surgery, for example, a robot will facilitate the surgical procedures, but they should be performed under the supervision of a human surgeon, who must have the capability to abort the robotic procedure immediately, if necessary.

In fact, those who fear AI simply fear a black box! In order to make a proper determination, we should know what methodologies are used exactly in the AI black box and how those methodologies are implemented and operated. So, we should explore the black box carefully and in detail (with the help of experts who are knowledgeable in the subject) and only then indicate what methodologies in the AI black box might be dangerous. Then other experts will be able to respond intelligently and in an informative manner.

\subsection{Characteristics of intelligence}

Before exploring AI itself, let us examine intelligence. No precise definition exists for intelligence. They are the external characteristics and capabilities (that we observe from actions) that enable us to claim whether an entity (for example, a robot) is "intelligent". Essentially, the outward characteristics define intelligence.

The characteristics of intelligence include sensory perception; pattern recognition; learning (i.e., knowledge acquisition, which is extremely important for intelligence); inference (i.e., making decisions) from incomplete information; inference from qualitative or approximate information (this is commonly used in "qualitative reasoning" as in fuzzy logic or fuzzy reasoning); ability to deal with unfamiliar situations; adaptability to new, yet related situations (through "expectational knowledge". For example, a human is able to expect the nature of an environment, like a classroom, even when encountering that environment for the first time); inductive reasoning (people must have done this in high school mathematics when proving a mathematical result "by induction"); common sense; display of emotions; inventiveness; and self-awareness (i.e., knowing their own capabilities).

A simplified model for the dynamics of intelligence is shown in Figure 4. The intelligent preprocessors are, in fact, learning modules. They enable one to gain knowledge by "learning" from information and also achieve expertise by further learning through knowledge (including practice). The achieved knowledge and expertise can depreciate for various reasons (including environmental and biological) and also can become outdated. Even though intelligent preprocessing or learning is vital in this model, it is unlikely that machine 


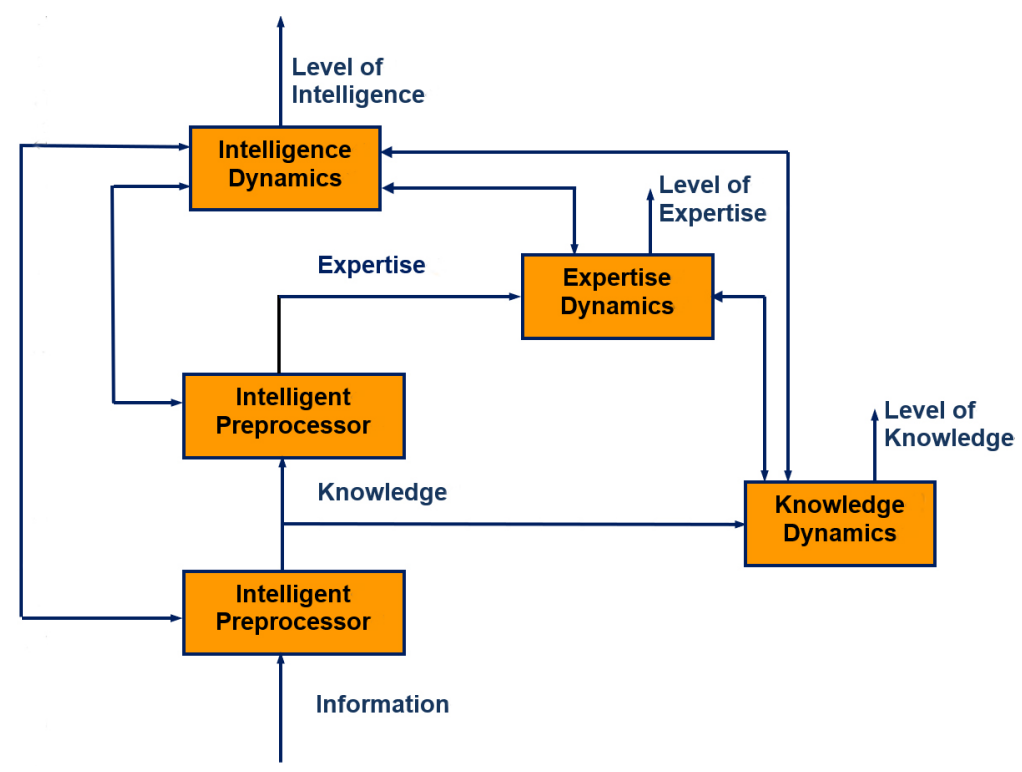

Figure 4. A model for the dynamics of intelligence.

learning alone will be able to achieve all forms of human intelligence in a robotic device.

\subsection{Artificial intelligence}

AI uses formal techniques to acquire some characteristics of intelligence. Models of AI are used for this purpose based on one or more of the mentioned characteristics. Such approaches (or models) of AI include Machine Learning, a very popular approach to AI. The conventional models of AI include knowledge-based systems, soft computing (consisting of neural networks - NN, fuzzy systems, and evolutionary computing; see $^{[g]}$ for instance), and swarm intelligence. A knowledge-based system typically consists of a knowledge base (or a rule base), a database, and an inference engine (the decision-maker). The decisions are made as follows: some data in the database (including what is acquired recently through sensors) is matched with the (context of the) rules in the knowledge base by the inference engine, and the inferences (or actions) are determined accordingly (i.e., rules are fired). Popular "Expert Systems" are based on this model. Of course, the knowledge base will be improved and enhanced continuously through "learning" and experience (so machine learning is used here as well).

Deep learning is a popular approach to machine learning. It incorporates an intelligent and intensive method of learning and sophisticated computing power that is available with such advancements as graphic processing units and tensor processing units to process massive quantities of data efficiently. Deep learning need not be limited to the use of deep NN but is the current trend. Deep NN includes Convolutional NN or convolutional neural network $(\mathrm{CNN})^{[4,10]}$ (see Figure 5). They have a structure of multiple layers (convolution layers) incorporating the "dynamic" learning ability and ending with a "Softmax" layer, which is the classification layer. First, the NN is trained using "labeled data" (i.e., input data whose proper outcomes are known a priori). Then, after the network is trained properly, unlabeled data (or new data) may be used for actual decision-making. Thus, massive amounts of data, including sensed data (a mixture of labeled and unlabeled data), may be effectively used in a deep NN.

Reinforcement learning relies on rewarding the correct decisions and penalizing the wrong decisions to learn the proper decision strategies. AI agents are capable of providing explanations for their decisions 


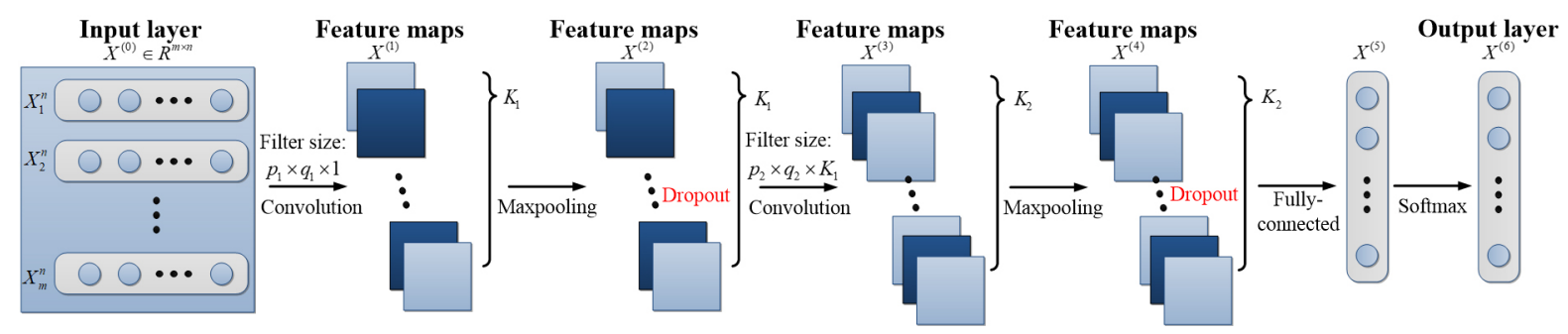

Figure 5. The architecture of a convolutional neural network.

(similar to what Expert Systems provide). In Edge AI, AI algorithms are processed locally on a hardware device. The algorithm uses data created on the device (e.g., data generated by the algorithm) and other data (external data, including those from sensors and through the system interface). Hence, Edge AI functions at the "edge of the system network". Fuzzy logic attempts to be similar to human decision-making by incorporating "fuzzy" or "qualitative" or "approximate" data, such as those that use qualifiers like fast, small, better, and close. Qualitative or fuzzy reasoning is used in the decision-making (inference) process. Swarm Intelligence behaves like a swarm of animals or insects. They are distributed (not hierarchical) and interact with each other to learn and make decisions. The members in a swarm use very simple rules, yet leading to "intelligent" global behavior, even though an individual member is not quite intelligent, which will improve with time. Evolutionary computing relies on genetic algorithms or genetic computing to realize "optimized" behavior through learning. The basis of this methodology is biological evolution (or survival of the fittest).

Within AI, apart from "learning", other characteristics of intelligence need to be investigated and incorporated (e.g., decision making with partial, approximate or qualitative information, use of "expectational knowledge", various approaches of reasoning such as inductive reasoning, ability to deal with unfamiliar situations, common sense, inventiveness, self-awareness, attention representation, and classification). Under machine learning itself, many methods exist, such as CNN, dynamic or recurrent neural networks (RNN), reinforcement learning, support vector machines, and entropy-based approaches. The rationalization of why a particular learning method is chosen (justification) should be a requirement in any application. Comparative evaluations of different methods should be carried out, with a proper reference (basis) for comparison. In this manner, comparative advantages and disadvantages of different methods should be determined, the rationale for choosing a particular approach for the application. When machine learning is applied in a particular situation, domain transformation or domain adaptability needs consideration because the domain of learning is typically not the same as the domain of application ${ }^{[11]}$.

\section{ROBOTIC CONTROL}

It should be clear that proper control techniques are crucial for the effective operation of a robotic system. Typically, different types of multiple robots are used in practical applications. Then, networked and automated or autonomous operation of multiple robots, in a common, self-adaptive, and intelligent system architecture, implemented on a common platform, with resource sharing, has to be implemented.

The networked operation of multiple robots and other agents (sensors, actuators, controllers, and other devices) is not new. Furthermore, system optimization, intelligent systems, and adaptive control have been extensively investigated and applied by us and others. In this backdrop and the strong foundation of prior work, the networked implementation of multiple robots (and other agents) may focus on the following aspects: 
1. Some networked agents (e.g., robots, unmanned aerial vehicles or UAVs, and sensor nodes containing sensors, actuators, effectors, controllers, etc.) may be dynamic or mobile.

2. The operating system environment may be dynamic, unstructured, and unknown.

3. The system should be self-adaptive to optimize its performance, particularly by utilizing the dynamic components in addition to parameter adjustment or tuning and structural reorganization.

4. The system may be further optimized by sharing resources among the applications.

5. Dynamic or mobile sensors may receive "feedback" from themselves to improve their sensing effectiveness (e.g., data/information quality, the relevance of their data, speed, and confidence).

6. The networked agents should possess "intelligence" to facilitate autonomous and desired performance.

7. The system should be able to predict, detect, and diagnose malfunctions and faults in it and accommodate or self-repair.

The underlying activities of system development and implementation will pertain to sensor/data fusion and adaptive sensing; multi-agent cooperation; multi-objective and parameter/structure optimization; fault prediction, detection, diagnosis, and resolution; self-organization/adaptation; and distributed/networked intelligent control. Suitable system architecture and an application platform of this type are schematically shown in Figure 6. In this system development process, one may have to determine and quantify the design constraints, performance limits, trade-offs, and development/operation guidelines and benchmarks for the pertinent applications. That will lead to significant improvements in performance, developmental and operational costs, productivity, resource requirements, energy efficiency, safety, fault tolerance, reliability, autonomy, and sustainability of the robotic system.

It is clear that both individual and network control are relevant in the present context, and furthermore, both conventional control and "intelligent" control are also relevant. The present paper has devoted much focus to the aspect of robotic intelligence. Hence, in the present section, particular attention is given to the conventional control of robots.

\subsection{Conventional control}

The development and application of conventional control have been extended wide effort by many. The relevant techniques include the following.

Feedback control and particularly servo-control of robotic joints had been the main focus in the early developments of robotic control. Here, the robot motions are measured (sensed) and used by the controller in feedback to move the robot in the desired manner. Thus, a robot is "servoed" along a specified motion trajectory through feedback control of the motion error using servo control. Notably, the subject of design and compensation or tuning of proportional-integral-derivative control has received adequate attention.

An image of an object is indeed a valuable source of information about that object. In this context, the imaging device is the sensor, and the image is the sensed data. Depending on the imaging device, an image can be many varieties such as optical, thermal or infrared, X-ray, ultraviolet, acoustic, ultrasound, etc. The image processing methods are rather similar among these imaging devices. For example, the digital camera is a very popular optical imaging device used in various engineering applications such as vision-guided robotics. Such operations as object recognition, pattern recognition and classification, abstraction, and knowledge-based decision making can be carried out using the information extracted through image processing. Visual servoing ${ }^{[12,13]}$, in particular, has received much attention and is commonly implemented in robotics. Here, the robot motion, including the actual position of the end effector (gripper, hand, tool, etc.) and the relative position of the targeted object, is measured using camera images and compared with 


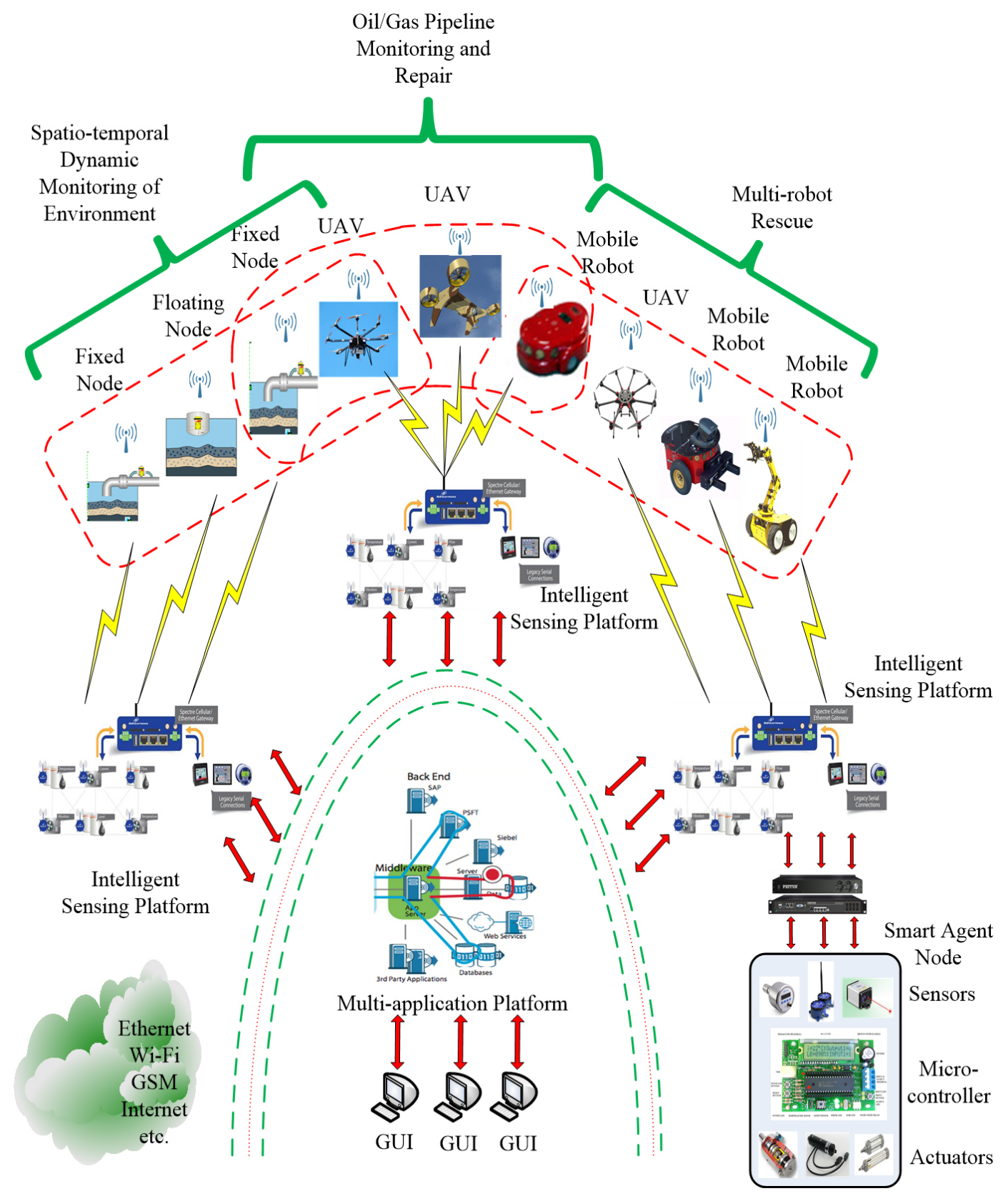

Figure 6. A multi-robot system layout. UAV: Unmanned aerial vehicle; GUl: graphical user interface; GSM: global system for mobile communication.

the desired or reference values. This difference (error) is used to generate a motion command (feedback control) for the robot so that the end effector would follow the desired trajectory and carry out the robotic task.

A robotic system may have inputs that do not participate in feedback control. These inputs are not compared with feedback (measurement) signals to generate control signals. Some of these inputs might be important variables, while others might be undesirable, such as external disturbances and noise. Generally, the performance of a robotic system can be improved by measuring these (unknown) inputs and using the information to generate the control actions. In feedforward control, unknown "inputs" are measured, and 
that information, along with desired inputs, is used to generate control signals that can reduce errors due to these unknown inputs or variations in them. The reason for calling this method feedforward control stems from the fact that the associated measurement and control (and compensation) take place in the forward path of the control system. Both feedback and feedforward schemes may be used in the same control system. In some robotic applications, control inputs are computed using the desired outputs and accurate dynamic models for the robots, and the computed inputs are used for control purposes. This is the "inverse model" (or "inverse dynamics") approach because the input is computed using the output and a model (inverse model). In some literature, this method is also known as feedforward control. To avoid confusion, however, it is appropriate to denote this method as computed-input control.

Since the overall response of a plant (e.g., a robot) depends on its individual modes, it should be possible to control a robot by controlling its modes. This is the basis of modal control. A mode is determined by the corresponding eigenvalue and eigenvector. In view of this, a popular approach of modal control is the pole placement or pole assignment. In this method of controller design, the objective is to select a feedback controller that will make the poles of the closed-loop system take up a set of desired values. This approach uses a "linearized" model of the robot.

As we saw, a robot can be controlled using a feedback control law so as to satisfy some performance requirements. In optimal control, the objective is to optimize a suitable objective function (e.g., maximize a performance index or minimize a cost function) by using an appropriate feedback control law ${ }^{[14]}$. A particularly favorite performance index is the infinite-time quadratic integral of the state variables and input variables, and popular control law is linear constant-gain feedback of the system states. The associated controller is known as the linear quadratic regulator (LQR). Linear Quadratic Gaussian (LQG) Control is an optimal control technique that is intended for a linear system with random input disturbances and output (measurement) noise. An LQR controller together with a Kalman filter is used in this approach.

For servo control to be effective, nonlinearities and dynamic coupling of the robot must be compensated faster than the control bandwidth at the servo level. One way of accomplishing this is by implementing a linearizing and decoupling controller inside the servo loops. This technique is termed feedback linearization technique.

An adaptive control system is a feedback control system in which the values of some or all of the controller parameters are modified (adapted) during the system operation (in real-time) on the basis of some performance measure when the response (output) requirements are not satisfied. Many criteria can be employed for modifying the parameter values of a controller. Self-tuning control falls into the same category. Model identification or estimation may be required for adaptive control, which may be considered to be a preliminary step of "learning". A neural network may be used for this purpose. In a learning system, control decisions are made using the cumulative experience and knowledge gained over a period of time. Furthermore, the definition of learning implies that a learning controller will "remember" and improve its performance with time. This is an evolutionary process that is true for intelligent controllers but not generally for adaptive controllers. In model-referenced adaptive control, the same reference input that is applied to the physical system is applied to a reference model as well. The difference between the response of the physical system and the output from the reference model is the error. The ideal objective is to make this error zero at all times. Then the system will perform just like the reference model. The error signal is used by the adaptation mechanism to determine the necessary modifications to the values of the controller parameters in order to achieve this objective. 
Sliding mode control ${ }^{[15]}$, variable structure control, and suction control fall within the same class of control techniques and are somewhat synonymous. The control law in this class is generally a switching controller. A variety of switching criteria may be employed. Sliding mode control may be treated as an adaptive control technique. Because the switching surface is not fixed, its variability is somewhat analogous to an adaptation criterion. Specifically, the error of the plant response is zero when the control falls on the sliding surface.

$H \infty$ (H-infinity) control is an optimal control approach, which is different from the LQG method. This frequency-domain technique assumes a linear plant with constant parameters, which may be modeled by a transfer function (matrix in the general case). The underlying design problem is to select a suitable controller that will result in the required performance of the system. In other words, the closed-loop transfer matrix must be properly "shaped" through an appropriate choice of the controller. Specifically, the controller that minimizes the " $H \infty$ norm" of the closed-loop transfer matrix, which is the maximum value of the largest singular value of this matrix, is used.

For complex multi-robot systems having various and stringent operating requirements, distributed and networked control is appropriate. It may consist of many programmable logic controllers and a supervisory controller, which will supervise, manage, coordinate and control the overall system. In hierarchical control, the distribution of control is provided both geographically and functionally. The management decisions, supervisory control, and coordination between robots may be provided by the supervisory controller, which is at the highest level of the hierarchy. The next lower level may generate control settings (or reference inputs) for each control region (subsystem). Finally, setpoints and reference signals are inputs to the direct controllers of the robots. In master-slave distributed control, only downloading of information is available.

\subsection{Intelligent control}

In intelligent control, an "intelligent" method of decision-making is used to make the control decision (i.e., to generate the control action). Soft computing, consisting of neural networks, fuzzy systems, evolutionary computing, and even probabilistic methods, has been popularly used in intelligent control. The topic of soft computing has already been addressed under the general theme of the present paper and is not repeated here. However, it is adequate to mention that, since learning control is used in robotic control, any approach of machine learning such as deep learning and deep neural networks, as discussed earlier in the paper, maybe incorporated into intelligent control of robots.

\section{OPPORTUNITIES OF ROBOTICS}

The commercial applications of Intelligent Robotics (with AI) include: autonomous agents such as selfdriving vehicles (encompassing aerial, ground-based, and underwater vehicles), which are indeed mobile robots; assistive devices (active and adaptive prostheses, wearables, and hand-held smart devices); advisory systems (or, expert systems, which are used in such areas as medical, legal, business, service, and social); monitoring/security systems (they are applicable in such areas as machine fault detection, prediction and diagnosis; and for human health monitoring, in telemedicine, homecare, etc.; video analysis; cyber security; human-machine interaction (including natural language processing, facial expression detection, speech recognition, communication, and intelligent connectivity; industrial application (including manufacturing and the assessment of production quality, cost, and efficiency); consumer, service, and entertainment sectors (retail, domestic, social, etc.); agriculture (growing, fertilizing, weed removal, and harvesting); smart buildings (heating ventilation, and air conditioning - HVAC; smart metering, safety, smart appliances, automated lighting, and achieving energy efficiency); education ("intelligent" learning management system or LMS, collaboration among students and with teachers - this approach may be quite beneficial in the current epidemic situation of Covid-19); and energy and environment (distribution, exploration, 
monitoring, planning, and utilization of energy). Some of these applications have been implemented today. However, some will provide diverse future opportunities.

\subsection{Opportunities for developing countries}

Here, opportunities exist in all the areas that were mentioned before. However, developing countries should not blindly decide on the considered robotics activities just for the sake of being involved in Robotics or AI. It is important to explore and determine what is in the "black box". Otherwise, one can be dissuaded through fear-mongering or make wrong choices for robotic activities. One must first question whether Robotics is needed for a specific local application. Then they must explore which robotic approaches are relevant for the considered task. Very importantly, they must examine what is in the existing Black Box before implementing it.

Developing countries should primarily concentrate on "robot development", not their application for the automation of local industries. They will be able to market these robots to other countries. Since the developing countries typically have an excessive and smart labor force, using robots for such applications as agriculture and industrial automation is not generally suitable in those countries. Nevertheless, they may consider the development of simple and low-cost robots for local use (e.g., for service and household applications). They may focus on the development of advanced software, in particular, to incorporate other forms of intelligence into robots and efficient software, and the use of advanced platforms like Flexible Cloud, Real-time Internet of Things, and Edge AI. Software development can be carried out without much capital investment, as it is done in India particularly because these countries normally have an educated and vast group of professionals. Also, they should focus on advancing the "mechanical capabilities" of robots, which are essential but may not necessarily be for the local market. As well, they should consider the needs that result from a particular situation (e.g., Covid-19). Very importantly, they should develop their own guidelines and regulations for robotic ethics and safety, which can be done by modifying the existing guidelines and regulations in the highly developed jurisdictions.

\section{CONCLUSIONS}

Robotics has found numerous practical applications today in industry, medicine, the service sector, household, and the general society. Important developments and practical strides are being made, particularly in Soft Robotics, Mobile Robotics (Aerial - drones, Underwater, Ground-based - autonomous vehicles in particular), Swarm Robotics, Homecare, Surgery, Assistive Devices, and Active Prosthesis. This perspective paper presented a brief history of Robotics while indicating some associated myths and unfair expectations. Next, it presented some important practical applications of Robotics, as developed by groups worldwide, including the Industrial Automation Laboratory at the University of British Columbia, headed by the author. The main shortcomings of Intelligent Robotics included those of the mechanical capabilities and the nature of the available level of intelligence. Concerning robotic intelligence, apart from the current focus of "learning", other characteristics should be further explored and incorporated. They included sensory perception, pattern recognition, decision making from incomplete information, inference from qualitative or approximate information (qualitative reasoning), ability to deal with unfamiliar situations, adaptability to new, yet related situations (through "expectational knowledge"), inductive reasoning, common sense, display of emotions, inventiveness, and self-awareness. Finally, the future trends and key opportunities available in Intelligent Robotics for both developed and developing counties were indicated.

\section{DECLARATIONS}

\section{Authors' contributions}

The author contributed solely to the article. 


\section{Availability of data and materials}

Not applicable.

\section{Financial support and sponsorship}

This work has been supported through research grants from the Natural Sciences and Engineering Research Council (NSERC) of Canada.

\section{Conflicts of interest}

The author declared that there are no conflicts of interest.

\section{Ethical approval and consent to participate}

Not applicable.

\section{Consent for publication}

Not applicable.

\section{Copyright}

(c) The Author(s) 2021.

\section{REFERENCES}

1. Unimate, the first industrial robot. Available from: https://youtu.be/hxsWeVtb-JQ?t=22. [Last accessed on 9 Aug 2021].

2. Welding robots in an automotive plant. Available from: https://youtu.be/0L7Xk5_s3QQ. [Last accessed on 9 Aug 2021].

3. Humanoid robot, Honda Asimo. Available from: https://asimo.honda.com/. [Last accessed on 9 Aug 2021].

4. Chen J, Shu T, Li T, de Silva CW. Deep reinforced learning tree for spatiotemporal monitoring with mobile robotic wireless sensor networks. IEEE Trans Syst Man Cybern, Syst 2020;50:4197-211. DOI

5. Li T, Wang C, Meng MQH, de Silva CW. Coverage sampling planner for UAV-enabled environmental exploration and field mapping. Proceedings of the IEEE/RSJ International Conference on Intelligent Robots and Systems (IROS); 2019 Nov 3-8; Macau, China. 2019. DOI

6. Zhang K, Luo J, Xiao W, et al. A subvision system for enhancing the environmental adaptability of the powered transfemoral prosthesis. IEEE Trans Cybern 2021;51:3285-97. DOI PubMed

7. de Silva CW. Video interview on the award of Killam Research Prize, The University of British Columbia, Vancouver, Canada. Available from: https://youtu.be/rMW32o2QD5E. [Last accessed on 9 Aug 2021].

8. Time delay in teleoperation. Available from: https://youtu.be/sl5ckyY7Zao?t=9. [Last accessed on 9 Aug 2021]

9. Karray FO, de Silva CW. Soft computing and intelligent systems design: theory, tools, and applications. Addison Wesley, New York, NY. 2004.

10. Xia M, Li T, Xu L, Liu L, de Silva CW. Fault diagnosis for rotating machinery using multiple sensors and convolutional neural networks. IEEE/ASME Trans Mechatron 2018;23:101-10. DOI

11. Wang J, Chen J, Lin J, Sigal L, de Silva CW. Discriminative feature alignment: improving transferability of unsupervised domain adaptation by Gaussian-guided latent alignment. Pattern Recogn 2021;116:107943. DOI

12. Zhang Y, Wang Y, Lang H, Wang Y, de Silva CW. Visual avoidance of collision with randomly moving obstacles through approximate reinforcement learning. Instrumentation 2019;6:59-66. DOI

13. Wang Y, Zhang G, Lang H, Zuo B, de Silva CW. A modified image-based visual servo controller with hybrid camera configuration for robust robotic grasping. Robot Auton Syst 2014;62:1398-407. DOI

14. Ma J, Cheng Z, Wang W, et al. Convex inner approximation for mixed H2/H-infinity control with application to a 2-DoF flexure-based nano-positioning system. IEEE Trans Ind Electron 2021. DOI

15. Wang W, Ma J, Cheng Z, Li X, de Silva CW, Lee TH. Global iterative sliding mode control of an industrial biaxial gantry system for contouring motion. Available from: https://arxiv.org/abs/2103.12580 [Last accessed on 9 Aug 2021]. 